LIAMES 5 - pp. 7-24, Primavera 2005

\author{
Alejandra Vidal \\ (Conicet-Universidad Nacional de Formosa) \\ Verónica Nercesian \\ (Facultad de Filosofía y Letras, UBA)
}

\title{
Sustantivos y verbos en wichí: hacia una taxonomía de clases de palabras ${ }^{1}$
}

\begin{abstract}
This work provides an analysis of the distinction between 'noun' and 'verb' in Wichí (mataco-mataguayan) on the basis of morphological and syntactic criteria. The authors argue that despite formal and functional similarities (noun and verb share certain affixes and enclitics, morphological nouns behave as predicates) the distinction between the two classes is robust. In addition, this article discusses the noun and the verb templates and their position classes. It is organized into six sections. Section 2 introduces some typological features of the language. The working hypothesis is provided in section 3. Section 4 focuses on the formal properties of nouns and verbs. Section 5 attempts to compare both word-classes; showing differences and similarities between them. Conclusions are given in section 6 . Data come from the Teuco variety spoken in the provinces of Formosa and Chaco, in Argentina.
\end{abstract}

KEYWORDS: South America; Mataco-Mataguayan; Noun; Verb; Word classes; Morphosyntax.

RESUMEN: El presente trabajo se propone discutir acerca de la diferencia entre 'sustantivo' y 'verbo' en wichí, una lengua de la familia mataco-mataguaya, sobre la base de criterios morfológicos y sintácticos. Las autoras sostienen que a pesar de la similitud formal y funcional de ambas clases (comparten afijos y enclíticos, los sustantivos pueden ocasionalmente cumplir funciones de predicado) la diferencia entre 'sustantivo' y 'verbo' en dicha lengua es robusta. Además, este estudio aborda la organización interna del sustantivo y del verbo. El mismo se organiza en seis partes. En la sección 2, se presentan algunos rasgos generales de la lengua. En 3, la hipótesis de trabajo. La parte 4 está dedicada al tratamiento de las clases léxicas mayores (sustantivos y verbos). En 5 se desarrolla las semejanzas y diferencias entre ambas clases de palabras. Las conclusiones se presentan en 6. Los datos corresponden a la variedad del Teuco (zona del río Bermejo) hablada en las provincias de Chaco y Formosa, en Argentina.

PALABRAS-CLAVE: Sudamérica; Mataco-Mataguaya; Sustantivo; Verbo; Clases de palabras; Morfosintaxis.

\footnotetext{
${ }^{1}$ El trabajo fue realizado en el marco del proyecto "Lenguas en peligro, pueblos en peligro en Argentina" dirigido por la Dra. Lucía Golluscio, FFyL-UBA, en convenio con el Max Planck Institute for Evolutionary Anthropology (Leipzig). Los datos sobre los cuales se trabajó fueron relevados en las provincias de Formosa y Chaco, y corresponden a la variedad dialectal de la zona del río Bermejo. Agradecemos especialmente a Francisco Palacios, Severiano Bonilla, Francisco López, Alberto González, Gustavo Hilario y Daniel López, por su colaboración y trabajo en la elicitación de los datos.
} 


\section{INTRODUCCIÓN}

En lingüística, los estudios tipológicos en el siglo XX asumen que cada lengua tiene un inventario propio de clases de palabras que hay que definir. Por lo tanto, y en línea con aquellas discusiones, el estudio del wichí nos plantea los siguientes interrogantes: cuáles son las categorías básicas que debemos establecer a fin de poder realizar generalizaciones sobre las clases léxicas en esa lengua y cuáles las particularidades morfosintácticas de dichas clases. El presente trabajo sugiere algunas respuestas alrededor de dichas preguntas.

En wichí, se advierten dos clases mayores de palabras: los sustantivos y los verbos, que suelen ser complejos, hasta compuestos por varias raíces a las cuales se le pueden agregar clíticos y afijos. El objetivo de este trabajo es entonces, definir formal y funcionalmente las dos clases léxicas mayores, discutiendo a su vez semejanzas y diferencias entre ambas. En última instancia, esperamos brindar elementos para la construcción de una taxonomía de clases de palabra en las lenguas del Chaco Meridional.

Este trabajo se organiza de la siguiente manera. En §2, presentamos generalidades sobre la lengua y sus hablantes. En $§ 3$, planteamos nuestra hipótesis de trabajo. §4 está dedicada al tratamiento de las clases léxicas mayores. En $\$ 5$ desarrollamos las semejanzas y diferencias entre ambas clases. Las conclusiones se presentan en $§ 6$.

\section{LA LENGUA Y SUS HABLANTES}

En este apartado, nos referiremos a la ubicación geográfica del pueblo wichí, la cantidad de hablantes y la filiación genética de la lengua. A continuación, haremos una síntesis de las características morfosintácticas más salientes.

\subsection{Ubicación geográfica y filiación lingüística}

El wichí es una lengua de la familia mataco-mataguaya hablada en Argentina y Bolivia. En la Argentina, los wichí se encuentran en las provincias de Chaco, Salta y Formosa. En Bolivia, están ubicados en el departamento de Tarija, en la frontera entre Bolivia y Argentina. El número exacto de hablantes es incierto. García (2002) menciona la existencia de 40000 hablantes wichí sólo en Argentina. Además, según este autor, antropólogos y lingüistas han advertido en los últimos años, que los wichí conforman un conglomerado étnico que encierra mayores diferencias lingüísticas y culturales de las que da cuenta la clasificación tradicional en tres parcialidades (nocten -Yacuiba, Villa Montes y Crevaux, en Bolivia-, vejoz -área comprendida entre la ciudad de Tartagal, en Salta, y el río Bermejo- y guisnay -ribera derecha del río Pilcomayo en las cercanías de la frontera entre Argentina y Bolivia). En el plano de la lengua, para Braunstein (1992-93), los asentamientos se disponen como un conjunto de unidades discretas y contiguas de variación constante, a la manera de una "cadena dialectal". Mientras que los sujetos que pertenecen a dos unidades próximas logran comunicarse en forma fluida, aquellos que provienen de unidades muy distantes geográficamente, deben utilizar el español como lengua franca. Las diferencias culturales, en alguna medida, parecen seguir el mismo patrón que las variedades lingüísticas. 


\subsection{Características morfosintácticas}

A causa del número de categorías gramaticales y de la variación morfológica que pueden manifestar, el verbo devela una complejidad superior a la de ninguna otra clase de palabra.

No existe la marcación de caso para los sustantivos. Por el contrario, todos los roles aparecen indicados en el verbo, característica que acerca al wichí al tipo de lengua de marcación en el núcleo ('head marking'), con tendencia a la estructura polisintética. ${ }^{2}$

Las categorías de negación, persona (sujeto TR/INTR), tiempo, aspecto, locación/direccionalidad se manifiestan en el verbo. Además, existen sufijos asociados a la transitividad, que además de alterar la relación entre el verbo y sus argumentos, acarrean cambios para el significado de la raíz (por ejemplo, w'en "ver, tener" w'enhu "dar"). Éstos ocurren solos o en combinación con sufijos pronominales de objeto (por ejemplo, w'en'uhu "me da"). Al igual que los afijos de valencia, los de locación y dirección también pueden derivar nuevos significados a partir del significado de la base: (t'eye "mirar" versus t'ekwe "buscar").

Desde el punto de vista sincrónico, la lengua es del tipo nominativo-acusativo: una misma serie de prefijos de sujeto se utiliza con verbos transitivos e intransitivos, cualquiera sea el rol semántico de aquél ('agente', 'paciente' o 'experimentante').

La lengua tiene además un segundo paradigma para indexicalizar al objeto (semánticamente 'paciente', o 'beneficiario' de verbos transitivos); dichas formas aparecen después de la raíz. (cf. Cuadro 1)

\begin{tabular}{|c|c|c|c|c|}
\hline & & \begin{tabular}{|l|} 
SUJETO \\
Transitivo e \\
Intransitivo \\
\end{tabular} & OBJETO & POSEEDOR \\
\hline & Persona & Indic $\quad$ Imp & & \\
\hline & 1 & $\begin{array}{l}\text { n'- } \\
\text { nt- }\end{array}$ & -n'u & $\begin{array}{l}\text { n'- } \\
\text { nt- }\end{array}$ \\
\hline & 2 & $\begin{array}{l}\text { l(V)- } \\
\text { lat(V)- }\end{array}$ & $\begin{array}{l}\text {-'am } \\
\text {-’a }\end{array}$ & $\begin{array}{l}\text { ha- } \\
\text { a- }\end{array}$ \\
\hline Singular & 3 & $\begin{array}{l}\text { t(V)- } \\
\text { i- } \\
\text { hi- } \\
\text { ni- } \\
\text { Ø- }\end{array}$ & \begin{tabular}{|l|}
$-\varnothing$ \\
$-' e$
\end{tabular} & $\begin{array}{l}1(\mathrm{~V})- \\
\varnothing-\end{array}$ \\
\hline & 3 (indet.) & $\begin{array}{l}\text { to- } \\
\text { ti- }\end{array}$ & & $\begin{array}{l}\text { to- } \\
\text { tot- }\end{array}$ \\
\hline
\end{tabular}

\footnotetext{
${ }^{2}$ Esto significa que el verbo wichí equivale a una cláusula, pues todos los argumentos se manifiestan en él.
} 


\begin{tabular}{|c|c|c|c|c|c|}
\hline \multirow{4}{*}{ Plural } & 1 & Inclusivo & $\begin{array}{l}\text { n'-(pl) } \\
\text { nt- (pl) } \\
\text { na- (pauc) } \\
\text { nat-(pauc) }\end{array}$ & \multirow[t]{2}{*}{-nam } & \multirow[t]{2}{*}{ lha- } \\
\hline & & Exclusivo & $\begin{array}{l}\text { to- } \\
\text { tot- } \\
\text { ti- }\end{array}$ & & \\
\hline & 2 & & $\begin{array}{l}\text { l(V)- } \\
\text { lat(V)- }\end{array}$ & -'am + -hen & $\begin{array}{l}\text { ha- } \\
\text { a- }\end{array}$ \\
\hline & 3 & & $\begin{array}{l}\mathrm{t}(\mathrm{V})- \\
\text { i- } \\
\text { hi- } \\
\text { ni- } \\
\emptyset-\end{array}$ & $-\varnothing+-h e n$ & $\begin{array}{l}1(\mathrm{~V}) \\
\emptyset-\end{array}$ \\
\hline
\end{tabular}

Cuadro 1. Afijos Pronominales de Sujeto, Objeto y Poseedor

En el sustantivo se indican la posesión, la dimensión (aumentativo/ diminutivo), el número, la distancia del referente con respecto a un centro deíctico, y su visualización por parte del enunciador. El género es, sin embargo, una categoría improductiva. La distinción masculino versus femenino sólo existe para unos pocos sustantivos del idioma, y se manifiesta a través de distintos sufijos. ${ }^{3}$ El paradigma de la persona del poseedor en los sustantivos es formalmente similar a la serie de prefijos de sujeto (cf. Cuadro 1).

\section{PLANTEO DEL PROBLEMA}

El primer problema que nos plantean los datos es la polisemia de algunos prefijos pronominales. La primera persona del singular se expresa a través de un mismo prefijo en el caso de los estados, los eventos y la posesión. Al respecto, véanse los ejemplos (1) donde $\underline{n^{\prime}-}$ puede indicar tanto al poseedor como al sujeto gramatical:

(1) a) n'kukahu

'Estoy limpio'

b) n't'ek

'Yo como'

c) n'w'et

'Mi casa'

\footnotetext{
${ }^{3}$ Es probable que se trate de antiguas clases nominales, hoy fosilizadas en algunos términos de parentesco wichí. Ejemplos de la distinción femenino masculino se observan en las siguientes palabras: n'chila (masc.) 'mi hermano', n'chita (fem.) 'mi hermana'; n'lhos (masc.) 'mi hijo', n'lhose (fem.) 'mi hija'.

Cuando se trata de animales, las diferencias de género no están marcadas morfológicamente sino por medio de los sustantivos atsinha ('hembra', en general 'fem') y asnak ('macho', en general masc.) (i.e. kaila atsinha 'chiva', kaila asnal 'chivo').
} 
Asimismo, las formas enclíticas no tienen un locus definido y por lo tanto, pueden ocurrir tanto con verbos como con sustantivos y otras clases de palabras. Este tema será desarrollado en \$5.1. (Sin embargo, en este trabajo mostraremos la ocurrencia con verbos y sustantivos exclusivamente, aunque eventualmente hagamos referencia a su uso en otros contextos).

El segundo problema surge del hecho de que algunas raíces están léxicamente indeterminadas. Esto implica entonces que tales raíces y/o temas (-'stems'- o compuestos de varias raíces) no pertenecerían a una clase morfosintáctica particular y por lo tanto, no estarían condicionadas a combinarse con afijos de una forma fonológica determinada. Este tema será tratado en $\$ 5.2$.

El tercer problema estriba en que también los sustantivos pueden funcionar como predicados. Esta ambigüedad funcional se relaciona con el primer problema, puesto que el prefijo de persona del poseedor es idéntico al de persona sujeto, hecho que no contribuye a la distinción entre predicados y argumentos. Este tema será tratado en $\S 5.3$.

Sin embargo - y a pesar de lo dicho - nuestra hipótesis es que la diferencia entre sustantivo y verbo existe en wichí. Intentaremos demostrar que las diferencias se asientan sobre bases morfológicas y sintácticas. Comenzaremos por discutir las diferencias morfológicas y luego, nos detendremos en las semejanzas (\$5) para presentar a continuación (§5.3), las que consideramos son diferencias a nivel sintáctico.

\section{MORFOSINTAXIS DE LAS CLASES MAYORES: SUSTANTIVO Y VERBO}

En wichí, los sustantivos presentan la siguiente estructura:

\begin{tabular}{|c|c|}
\hline Prefijos & $\begin{array}{l}\text { 1. Posesivo (cf. Cuadro 1) } \\
\text { 2. Posesión alienable/Clasificador posesivo }{ }^{4} \\
\text { ka- 'general', lo- 'animal' } \\
\text { 3. RAIZ O BASE }\end{array}$ \\
\hline Sufijos & $\begin{array}{l}\text { 4. Género (co-lexicalizado): -la 'fem', -ta 'masc'; - Ø 'masc', -e 'fem' } \\
\text { 5. Diminutivo-fwaj/Aumentativo -taj } \\
\text { 6. Número (-l, -lh,-lis, -s, -es, -as, -ai, -oi, -ei, -i) }\end{array}$ \\
\hline $\begin{array}{l}\text { Enclíticos } \\
\text { Post- } \\
\text { inflexionales }\end{array}$ & $\begin{array}{ll}\text { 7. Distancia } & \\
\text { témporo-espacial: } & \text {-na 'próximo',-tsu, 'cislocativo',-tsi 'translocativo', } \\
& \text {-naji 'tiempo lejano', -mathi 'tiempo pasado- } \\
& \text { indefinido', -pa 'desconocido-no a la vista' }\end{array}$ \\
\hline
\end{tabular}

Figura 1. Estructura morfológica del sustantivo

\footnotetext{
${ }^{4}$ Golluscio (1993) llamó a estas dos formas 'marcadores de aptitud para ser poseídos’.
} 
Según el tipo de sustantivo (alienable/inalienable), éste consiste mínimamente de una raíz o de una raíz más un prefijo posesivo. (Los posesivos fueron presentados en el Cuadro 1). En cuanto al género, se expresa a través de morfemas que sincrónicamente están fusionados con la raíz (al respecto, cf. Nota 3). Respecto al número, se advierte la existencia de una decena de alomorfos de plural, muchos de los cuales no están sujetos a condicionamiento fonológico alguno.

Las clases posicionales 7 y 8 merecen algunas aclaraciones. Como el resto de las clases $(1,2,4,5$ y 6$)$, los miembros de la clase 7 y 8 son prosódicamente dependientes. Cuando ocurren, el acento se desplaza a la última sílaba en consonancia con el modelo de acentuación wichí más recurrente ${ }^{5}$. Dicho de otro modo, los enclíticos de las posiciones 7 y 8 están fonológicamente integrados a la palabra. En este sentido, no se diferencian de los afijos. Y tampoco, al igual que los afijos, ocurren solos; por el contrario, son ligados. Los enclíticos de la clase 7 también ocurren con verbos ${ }^{6}$. La razón por la cual han sido ubicados fuera de la estructura básica del sustantivo (raíz + afijos) es porque estas formas no contribuyen ni a la flexión ni a la derivación de la palabra. Además, no sólo se advierten en combinación con sustantivos y verbos; también ocurren en la formación de otras clases de palabras $^{7}$. (Excepto - pa también incluido en la misma clase 7, que sólo ocurre en combinación con sustantivos).

Como adelantáramos en §2, los temas sustantivos están formados por una o dos raíces (cf. (2)). También pueden derivarse de una raíz verbal más un sufijo nominalizador (cf. (3)), o de un tema verbal compuesto por una raíz verbal y un sustantivo incorporado, el cual se "nominaliza" al recibir un marcador de posesión indeterminada to- (4):

(2)

a) alhe + poset 'ciempiés' (lit. 'pico de iguana')

iguana + pico

b) tshowet + lhos 'cría' (lit. 'hijo de un animal')

animal + hijo

c) lapes + lhele - y 'descendientes' (lit. 'últimos habitantes') último + habitante-PL

d) siputka + muk 'polenta' (lit. 'polvo de maíz') maíz + polvo

\footnotetext{
${ }^{5}$ En el léxico, aproximadamente entre un $80-90 \%$ de las raíces bisilábicas (sobre un total de 523 palabras relevadas), lleva acentuación en la última sílaba.

${ }^{6}$ En esos casos, si bien cargan un significado temporal, los matices semánticos son ligeramente diferentes (cf. §5.1).

${ }^{7} \mathrm{Al}$ respecto, véanse las siguientes formas:

$\begin{array}{lll}\text { (i) fwalanaji } & \text { fwala=naji } & \text { 'ayer' } \\ \text { (ii) fwalana } & \text { fwala=na } & \text { 'hoy' } \\ \text { (iii) tojtsu } & \text { toj=tsu } & \text { 'eso' } \\ \text { (iv) toja } & \text { toj=na } & \text { 'esto' }\end{array}$
}


e) to - fwefw + t'oj 'uña' (lit. 'cuero del dedo')

3POS.INDET - dedo+cuero

f) tot - kolo+ts'e 'pantorrilla' (lit. 'panza de la pata')

3POS.INDET-pata+panza

$(3)^{8}$

a) tochefwenyaj 'enseñanza'

to - chefwen - yaj

3POS.INDET-enseñar-NMZ

b) n'tichunhayaj 'mi pensamiento'

n' - tichunlhi - yaj

1POS-pensar-NMZ

(4)

a) tochemyenek 'ayudante'

to - chem + yenek

3POS.INDET-trabajar+persona

b) tofwatsenhat 'escupitajo'

to - fwatse $[\mathrm{n}]+[\mathrm{hu}] \mathrm{nh}$ at

3POS.INDET - escupir+ piso/tierra

c) totseknakat 'hebra/hilo de coser'

to - tsek + nakat

3POS.INDET - coser+hilo

\footnotetext{
${ }^{8}$ Los ejemplos se consignan en transcripción ortográfica. La ortografía utilizada en este trabajo corresponde a la propuesta del alfabeto wichí unificado. Esta ortografía es aceptada por los hablantes de la zona de donde provienen los datos. Las abreviaturas en los ejemplos corresponden a las siguientes categorías: ASP=aspecto; CAUS=Causativo; $\mathrm{CL}=$ clasificador posesivo; $\mathrm{COP}=$ cópula; $\mathrm{DEM} . \mathrm{COP}=$ demostrativo (cópula); DISTR=distributivo; ITER=iterativo; indet=indeterminado; N=Nominal; neg=negación; NMZ=nominalizador; OBJ=objeto paciente o benefactivo; PAS. CERC=pasado cercano; PAS.LEJ=pasado lejano; PL=plural; $\mathrm{POS}=$ posesivo; $\mathrm{POS}$.INDET=posesivo indeterminado; $\mathrm{PRO}=$ pronombre; $\mathrm{PROX}=$ próximo; $\mathrm{REL}=$ relacionante; $\mathrm{S}=$ sujeto; $\mathrm{SG}=$ singular; TRVZ=transitivizador; V=verbo; VBZ=verbalizador. El guión separa afijos entre sí, o afijos de raíces; '+' simboliza la unión entre raíces en el caso de las palabras compuestas; el signo "=" marca el límite entre las raíces y los enclíticos.
} 
Los sustantivos en wichí también pueden estar formados por una raíz más un morfema derivativo que se ha gramaticalizado completamente (véanse (5) y (6)): ${ }^{9}$

(5) [Raíz nominal + wu 'agente'] ( >wuye 'hacer')

a) mak - wu 'avispa' (lit. 'el hacedor de algo, de cosas')

b) pini - wu 'avispa de miel' (lit. 'el que hace miel')

(6) [Raíz nominal (poss) + hi 'lugar'] (>ihi 'estar')

a) to - fwes - hi 'guante' (lit. 'lugar de los dedos')

b) to - khoy - hi 'bolsillo' (lit. 'lugar de los accesorios')

Por otro lado, el verbo presenta una estructura morfológica bastante más compleja (cf. Figura 2):

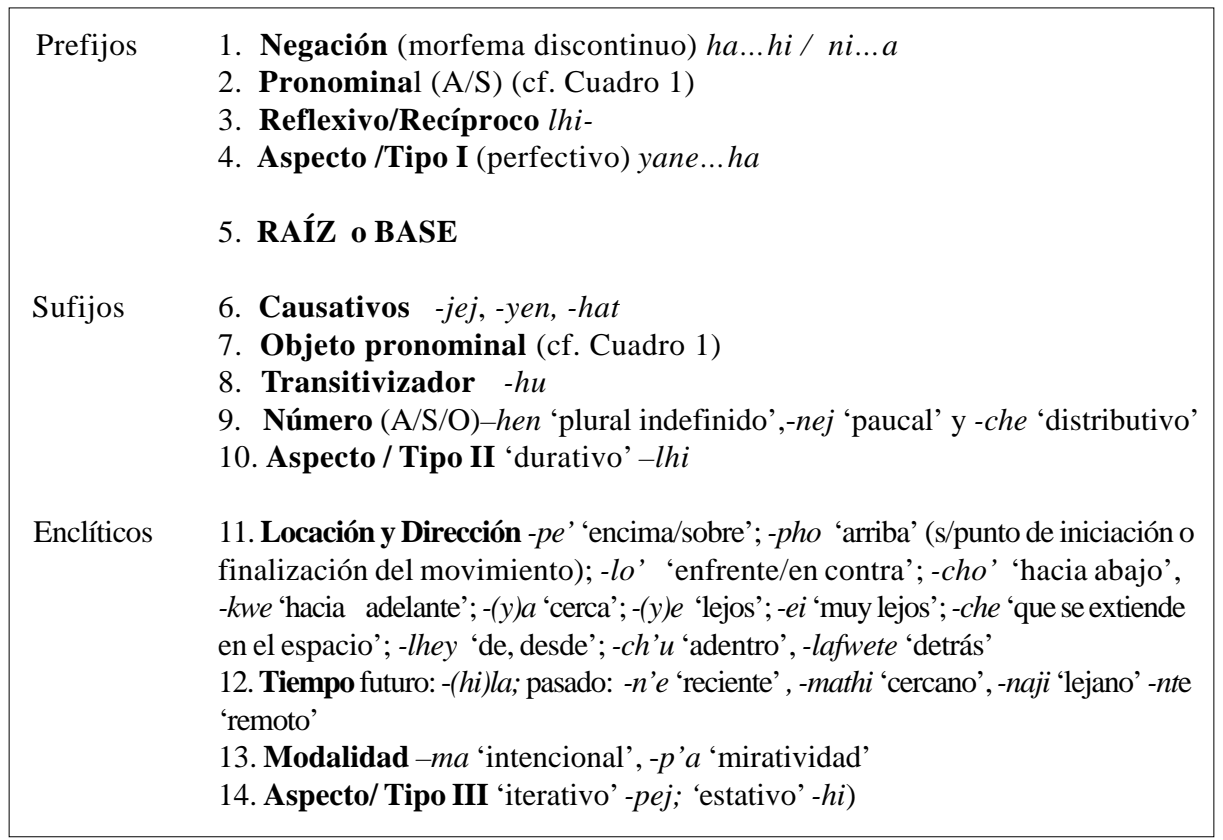

Figura 2. Estructura morfológica del verbo

\footnotetext{
${ }^{9}$ Ciertas formas léxicas han ido un paso más allá de la composición de raíces (cf. (2)), extendiéndose a través del léxico y generalizándose como marcadores gramaticales, a causa de la recurrencia de uso. Sin embargo, en (5) y (6) los sufijos derivativos provienen de un verbo y no un sustantivo como en (2).
} 
En cuanto a su estructura básica, excepto la raíz y el prefijo pronominal de sujeto, las demás posiciones no son obligatorias. Además, no todas van a ser "llenadas" al mismo tiempo; por ejemplo una forma verbal como n'-lhi-lej 'me lavo' no puede llevar el prefijo de reflexivo/recíproco lhi- y un transitivizador o un causativo simultáneamente. Si la intención es indicar que la acción de los participantes es recíproca, se combinará necesariamente con un sufijo de plural de la clase posicional 9, como en notshas lhiyainhen 'los chicos se miran entre sí'. Un prefijo reflexivo/recíproco no podría ocurrir tampoco con un sufijo pronominal de objeto. De esta manera, la combinación entre determinadas posiciones depende no sólo del significado que se quiera expresar, sino también de las restricciones de co-ocurrencia que pueden existir entre por ejemplo, los afijos de aumento/disminución de valencia, las distintas clases de verbos y el número de argumentos que rige, o entre las clases de verbos y los sufijos de aspecto. Al respecto, los siguientes verbos, por ejemplo, no admiten el aspectual -lhi 'durativo': n'yeneyche 'yo creo' *n'yeneychelhi; n'nom 'yo llego' *n'nomlhi; n'lota 'yo escucho' *n'lotahi/*n'lothi; n'yahin 'yo miro' *n'yahinhi. Parece que los llamados verbos de estado y logros (según la clasificación de aspecto léxico que establece Vendler, 1967), no se pueden combinar con -lhi, y los de percepción - al menos hasta el momento confirmados -, no se pueden combinar con -hi 'estativo'. Por el contrario, el sufijo de aspecto - $p e j$, parece ser mucho más productivo dado que se puede combinar con sustantivos y verbos (de estado, logros, efectuaciones y actividades) con valor distributivo y/o iterativo respectivamente (Sobre la ocurrencia de $-p e j$ con sustantivos y verbos, ver ejemplos en (10) más abajo).

Por otro lado, el verbo es la clase de palabra capaz de encodificar el lugar donde se inicia y transcurre el evento (locación), hacia donde éste se orienta (dirección), o el movimiento que recorre (trayectoria). Al respecto, véase en la Figura 2, la variedad de formas que ocupan la clase posicional 11.

Varias categorías de la Figura 2 están representadas por morfemas discontinuos. Sin embargo, no existen casos en que las categorías tengan que marcarse recursivamente. Así, la posición 9 será ocupada por un morfema que o bien señale el número de participantes comprendido en la clase sintáctica de 'sujeto' o bien del 'objeto' de un verbo transitivo pero nunca dos veces si se trata de un sujeto plural y un objeto plural.

Al igual que los sustantivos, los verbos pueden estar formados por una o dos raíces (ver (7)). La segunda es una raíz nominal incorporada junto con su marcador de posesión (7a-b). A esta combinación se le agrega un sufijo que señala de manera transparente la clase de palabra de la cual se trata ${ }^{10}$ :

\footnotetext{
${ }^{10}$ Nótese que en (7) se trata de verbos como 'hacer', 'ir', 'estar (bien)', que son básicos y frecuentes en todas las lenguas del mundo y por lo tanto, no sólo son los que presentan mayores irregularidades en sus paradigmas, sino también los que más fácilmente tienden a co-lexicalizarse con otras formas léxicas y/o gramaticales.
} 
(7)
a) iwulhosa
'Se embaraza'

$\mathrm{i}-\mathrm{wu}+$ lhos - a

3S-hacer+(su)hijo-VBZ

b) iwulheya 'Se llama'

$\mathrm{i}-\mathrm{wu}+$ lhey - ya

3S-hacer+(su)nombre-VBZ

c) iwutamsecha 'Descansa'

$\mathrm{i}-\mathrm{wu}+$ tamsek - ya

3S-hacer+descanso-VBZ

d) iwuch'efwaya 'Se casa'

$\mathrm{i}-\mathrm{wu}+\mathrm{ch}$ 'efwa - ya

3S-hacer+esposo/a-VBZ

e) isalhuhu 'Está correcto'

$\varnothing-$ is + alhu - hu

3S-estar bien, ser bueno+llanura-TRVZ

f) inealhuhu 'Corre en línea recta'

$\mathrm{i}-$ nek + alhu - hu

3S-ir+llanura-TRVZ'

\section{SUSTANTIVOS VIS À VIS VERBOS}

\subsection{Enclíticos verbales y nominales}

Como mencionamos en $\S 4$, la lengua wichí parece ser sensible a la distinción entre afijos y enclíticos. Algunos de los llamados 'enclíticos' (clase posicional 7/8 y 11/14; cf. Figura 1 y Figura 2, respectivamente) pueden ocurrir con un sustantivo o con un verbo. Un ejemplo lo constituyen las formas -naji y -mathi (Figura 1, clase 7 y las formas homófonas de la clase 12, Figura 2) incluidas en la clase de los morfemas post-inflexionales. Al respecto, véanse (8) y (9) donde en (8a) y (9a) ocurre con un verbo y en (8b) y (9b) con un sustantivo:

(8) -naji

a)

n'thateinaji chinaj toj n'lhukwey

n'-thatei-naji chinaj toj n'-lhukwey

1S-enviar-PAS.LEJ. plata REL 1POS-familia

'Yo envié plata a mi familia' 
b)

$$
\begin{aligned}
& \text {...tha ch'ojtoj n'yomey mak toj n'lhukweynaji yomeypej... } \\
& \text { tha ch'ojtoj n'-yomey mayek toj n'-lhukwe-i-naji Ø-yomey-pej } \\
& \text { y otra vez 1S-contar cosa REL 1POS-padre-PL-PAS.LEJ 3S-contar ITER } \\
& \text { 'Y otra vez cuento las cosas que mis padres siempre contaban' }
\end{aligned}
$$

(9) -mathi

a) lataw'elhumathi hal'o lata - w'elh - hu - mathi 'vos trepaste el árbol' $2 \mathrm{~S}$ - trepar - TRVZ-PAS.CERC árbo
b) hal'omathi icho' hal'o - mathi i - cho' árbol-PAS.CERC $3 \mathrm{~S}$ - quebrarse
'Aquel árbol se quebró'

Los enclíticos temporales al combinarse con sustantivos (cf. (9b)) señalan que el referente y no el evento está ubicado dentro de una dimensión temporal anterior ('el árbol de aquel tiempo, el árbol viejo, del tiempo pasado, entonces 'aquel árbol'). En (8) el sentido de -naji es 'los días pasados, los otros días', en un tiempo indefinido.

De manera similar, -pej ‘iterativo’ de la clase Aspecto/tipo III (Posición 8, en la Figura 1; Posición 14, en la Figura 2) puede ocurrir con verbos y sustantivos (cf. (10)). Cuando ocurre ligado a un sustantivo adquiere significado distributivo 'cada uno/a' (cf. (10b)):

(10) a) y'ajn'upej

$\varnothing$ - y'aj - n'u - pej

3S-golpear-1SG.OBJ-ITER

b) atsinhapej tachemhu atsinha-pej ta-chema-hu mujer-DISTR 3S-agarrar-TRVZ
'Me golpea reiteradas veces'

\author{
inot 'Cada mujer busca agua' \\ inot \\ agua
}

Nótese que tanto en el sustantivo como en el verbo, los enclíticos se ubican en las últimas posiciones (7/8, cf. Figura 1;11/14, cf. Figura 2). Además, no todos los enclíticos se comportan de la misma manera, algunos se ligan a varias clases de palabras y otros sólo a una (por ejemplo, - $p a$ 'no visual, desconocido' sólo ocurre con sustantivos, mientras que - pej 'iterativo' puede ocurrir con las dos).

\section{2. (In)determinación léxica de los temas}

Existen raíces cuyo marco de subcategorización no está determinado para ninguna de las clases mayores. No obstante, el wichí exhibe mecanismos para indicar la clase morfológica a la que pertenece la raíz. En (11) son los prefijos pronominal posesivo la- y de sujeto hi-, respectivamente, los que determinan la clase de palabra de que se trata 
(sustantivo y verbo, en cada caso). En (11) la distinción es directa, puesto que para la tercera persona los sufijos pronominales para sujeto y poseedor son diferentes. En (12), puesto que se trata de un sustantivo alienable, lo que determina la subcategorización de la raíz es la presencia/ausencia del clasificador posesivo para sustantivos alienables $k a$-(Posición 2 de la estructura morfológica, cf. Figura 1). En (13) la forma es verdaderamente ambigua fuera del contexto de la oración.

(11) ts'e 'panza'/'chupar, succionar'

a) lts'e hikw'esche takosley lhache hal'o

la-ts'e hi-kw'es-che ta-kosle-y $\quad \varnothing$-lhache hal'o

3POS-panza 3S-cortarse-PL 3POS-intestino-PL 3S-quedar árbol

... 'su panza se cortó y sus intestinos quedaron en el árbol...'

b) Maltin hits'e chupete toj lachinij kho

Maltin hi-ts'e chupete toj la-chinij Ø-kho

Martín 3S-chupar chupete REL. 3POS-hermano 3POS-pertenencia

'Martín chupa el chupete que es del hermano'

(12) y'eche 'camisa'/ponerse adentro'

a) y'eche hikw'es 'La camisa se descosió'

y'eche hi - kw'es

camisa $3 \mathrm{~S}$ - cortarse

b) y'eche lakay'eche 'Se pone su camisa.' (lit. 'Se mete en su camisa')

$\varnothing$ - y'eche la-ka-y'eche

3S-meterse 3POS-CL-camisa

(13) Ihey 'nombre'/'llamarse'

a) n'lhey Francisco 'Yo me llamo Francisco'

n' - lhey Francisco

1S-llamarse Francisco

b) n'lhey ihi 'Yo tengo nombre.' (lit. 'Hay mi nombre')

n' - lhey ihi

1POS-nombre haber

A partir de los ejemplos (11-13) se sigue que hay raíces o temas que al no estar subcategorizados para alguna de las dos clases pueden funcionar como argumentos o predicados. A veces la forma de los prefijos ayuda a determinar de qué clase de palabra se trata (como en (11). Otras, es indecible a menos que la veamos funcionando dentro de una oración, como en (12) y (13), o apliquemos pruebas sintácticas, tales como la negación 
(cf. §5.3). Cuando se trata de un sustantivo, la construcción sintáctica que éste forma es de posesión (véase n'lhok ihi 'yo tengo comida' similar a (13b) n'lhey ihi). Por el contrario, si se trata de una construcción como n'lhey requiere un complemento, es decir, el nombre propio ('Francisco' en este caso) para ser predicado. Por lo tanto, la única interpretación posible para n'lhey en (13b) es 'es mi nombre', puesto que se trata de una forma nominal.

En síntesis, si bien los prefijos pronominales de las tres series, en lo que respecta al singular son idénticos (cf. Cuadro 1 y §3), y esta identidad podría estar apuntando a la imposibilidad de distinguir un sustantivo de un verbo cuando la palabra está marcada para la primera persona, la lengua provee recursos morfosintácticos que permiten desambiguar los casos en que el mismo marcador se une a un sustantivo o a un verbo.

Como quedó demostrado, las semejanzas formales existen y sin embargo, éstas no nos impiden afirmar la existencia de dos clases mayores bien distintas, porque también hay desemejanzas formales. Por un lado, hay categorías gramaticales que se manifiestan de manera exclusiva con una y otra clase de palabra: éste podría ser criterio necesario y suficiente para sostener tal distinción. Sin embargo, también vimos que hay prefijos y enclíticos que pueden ligarse a ambas clases de la palabra por igual. Y más aún, que existen en la lengua raíces que no están subcategorizadas para ninguna clase léxica. Por lo tanto a continuación examinaremos las funciones del sustantivo y del verbo, a fin de determinar algunas diferencias entre ambas clases sobre la base de criterios sintácticos.

\subsection{Argumentos y predicados}

Cada clase sirve a funciones diferentes. Sintácticamente, los sustantivos son argumentos (cf. 14) y rara vez ocupan el lugar del predicado (cf. (16b)). En este sentido, la lengua no es ambigua con respecto a si estamos frente a un verbo o a un sustantivo:

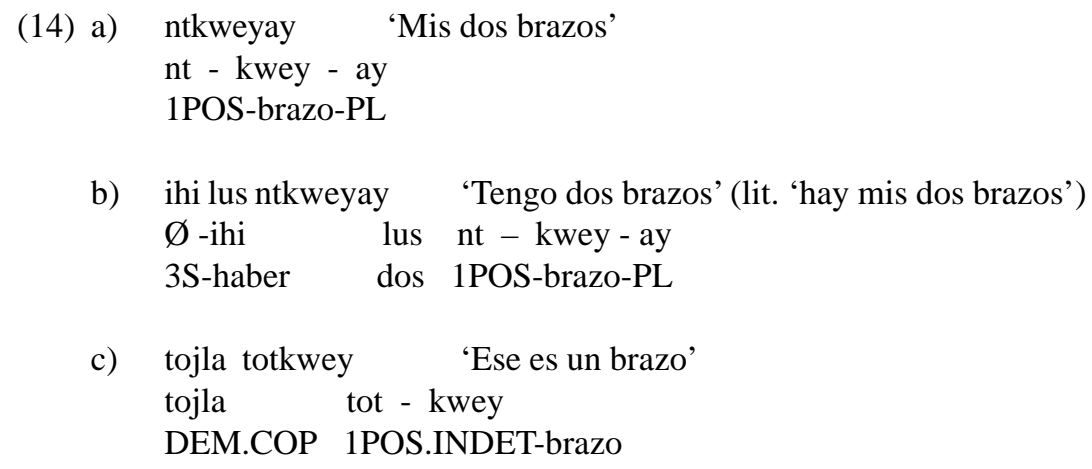

En (14) vemos tres estructuras gramaticalmente posibles, una sin verbo, una con verbo, y la tercera en la cual se advierte un sustantivo acompañado de una cópula. En ninguno de los tres casos la raíz sustantiva + el prefijo pronominal figuran en lugar del predicado oracional (especialmente en (14c), donde por su carácter de sustantivo inalienable, kwey 'brazo' lleva un prefijo posesivo necesariamente). 
Consideremos ahora los ejemplos en (15). Con referencia a los sustantivos alienables, en ninguna circunstancia un hablante usaría exclusivamente el pronombre de primera persona n' y la raíz (a)sinoj 'perro' para decir "mi perro" (15c). Los sustantivos alienables necesitan del clasificador para ser poseídos, en este caso lo- 'animal' (15a-b):

(15) a)

$$
\begin{aligned}
& \text { (n'lham) n'hope asinoj 'Soy perro' } \\
& \text { n'lham n' - hope asinoj } \\
& \text { PRO.1SG. 1S - ser perro }
\end{aligned}
$$

b) n'asinoj 'Soy un perro'

n' - asinoj

$1 \mathrm{~S}$ - ser perro

c) n'asinoj *'Mi perro'

Un caso idéntico se presenta en (16). No hay otra función para mayistalu que la de argumento del verbo hope 'ser'. Tanto (16a) como (16b) son posibles; (16b) es un predicado; en (16a) el sustantivo es el complemento del verbo hope. Sin embargo, (16c) donde el prefijo pronominal es interpretado como poseedor y no como argumento está excluida; es imposible que se use como construcción posesiva cuando el sustantivo es alienable:

(16) a) (n'lham) n'hope mayistalu 'Soy maestro'

n'lham n'-hope mayistalu

1S 1S-ser maestro

b) n'mayistalu 'Soy maestro'

n' - mayistalu

$1 \mathrm{~S}$ - maestro

c) n'mayistalu * 'Mi maestro'

En efecto, para que la primera persona sea entendida como el poseedor del referente debe intervenir el clasificador posesivo entre el prefijo posesivo y la raíz. Las oraciones $(17 \mathrm{c}-\mathrm{d})$ confirman que la presencia de los clasificadores $k a$ - y lo- para sustantivos alienables es necesaria para formar construcciones posesivas:

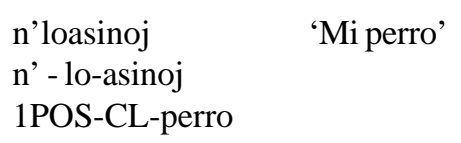

b) n'kamayistalu 'Mi maestro' n' - ka - mayistalu

1POS-CL-maestro 

c) n'asinoj
* 'Mi perro'
d) n'mayistalu
* 'Mi maestro'

Los sustantivos alienables funcionan como predicados cuando son usados con el prefijo pronominal más la raíz exclusivamente, sin que intervenga el clasificador posesivo. Por el contrario, los sustantivos inalienables siempre funcionan como argumentos y llevan la marca del poseedor.

El sustantivo inalienable precedido por el prefijo posesivo más la raíz nominal puede ser el argumento del verbo transitivo w'en 'tener', una forma no defectiva con conjugación completa:
(18) a) n'w'en n'kuset 'Yo tengo un pantalón'
n' - w'en n' - kuset
1S-tener 1POS-pantalón

b) n'w'en alhamis 'Yo tengo tu collar'

n' - w'en a - lhamis

1S-tener 2POS-collar

En suma, la lengua wichí tiene recursos en el nivel sintáctico para diferenciar sustantivos de verbos en casos de posible ambigüedad semántica. Todas esas estructuras señalan la función de argumento que desempeña el sustantivo. Una, consiste en el uso de un marcador posesivo para sustantivos alienables. Otra, en el uso del verbo 'tener', que admite flexión de persona y que al no ser una cópula, recibe un prefijo pronominal de manera obligatoria.

Segundo, todos los verbos admiten la negación. Al respecto, (19) ofrece ejemplos de construcciones negativas con verbos de existencia y posesión:

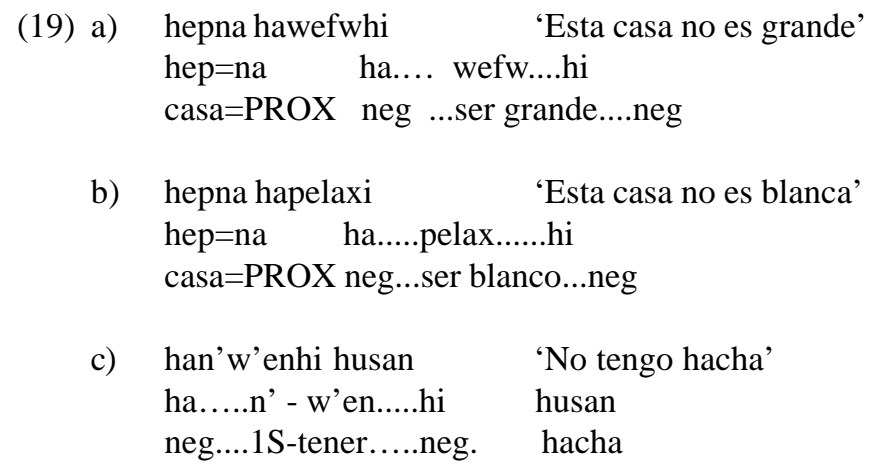

Sin embargo, también es posible que el sustantivo aparezca negado. En dicho caso, el sustantivo no funciona como argumento de un verbo sino como núcleo de una predicación posesiva (predicado de no verbal). Éste es el caso contrario al que se mostró en (14) y (15) más arriba, donde el sustantivo figuraba como argumento de los verbos hope 'ser' o ihi 'estar/haber'. 
En este sentido, (20) contrasta ejemplos de oraciones posesivas negadas con y sin verbo.

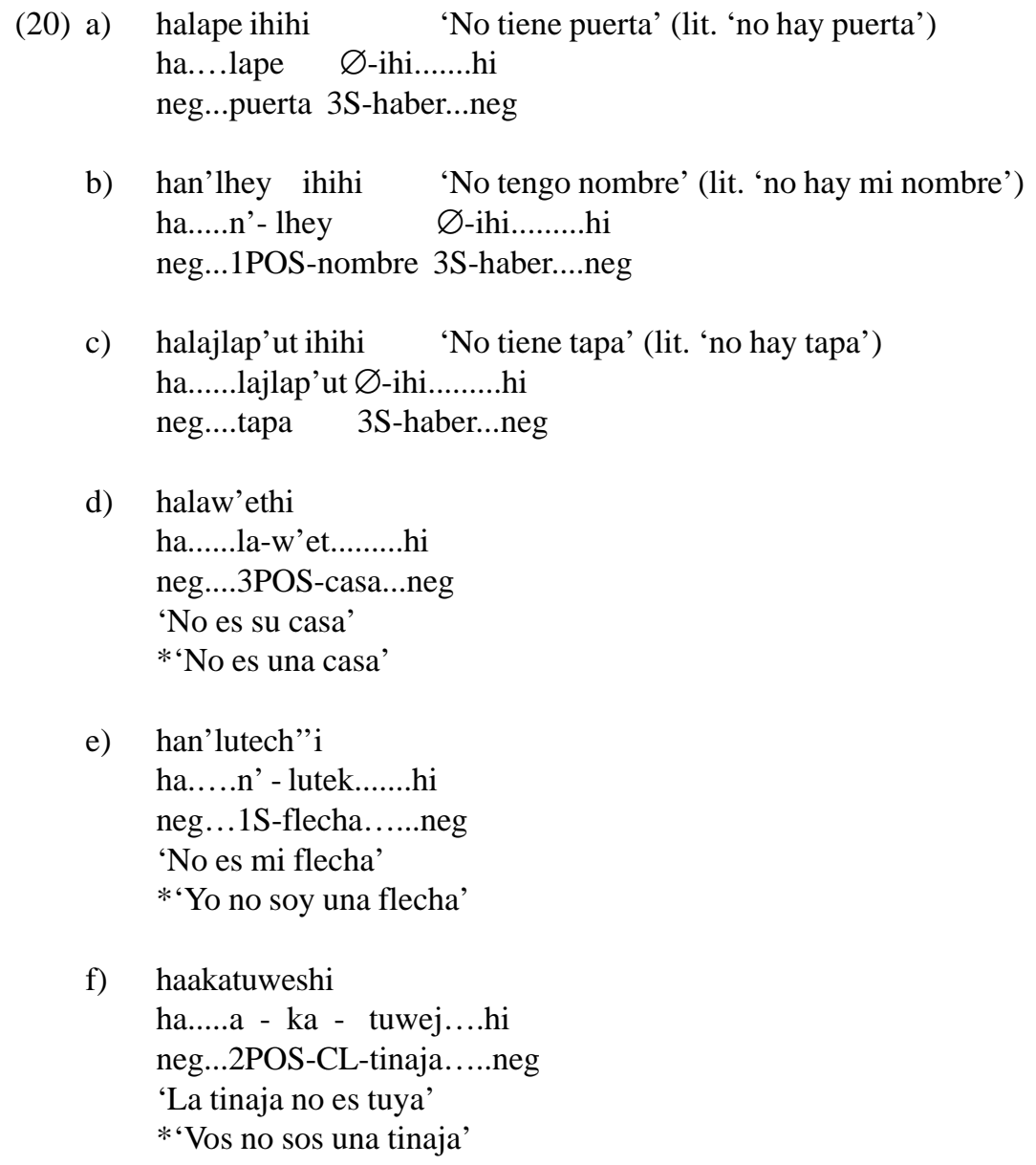

b) han'lhey ihihi 'No tengo nombre' (lit. 'no hay mi nombre')

ha.....n'-lhey $\varnothing$-ihi.........hi

neg...1POS-nombre 3S-haber....neg

c) halajlap'ut ihihi 'No tiene tapa' (lit. 'no hay tapa')

ha......lajlap'ut $\varnothing$-ihi.........hi

neg....tapa 3S-haber...neg

d) halaw'ethi

ha......la-w'et.........hi

neg....3POS-casa...neg

'No es su casa'

*'No es una casa'

e) han'lutech"i

ha.....n' - lutek.......hi

neg...1S-flecha......neg

'No es mi flecha'

*'Yo no soy una flecha'

f) haakatuweshi

ha.....a - ka - tuwej...hi

neg...2POS-CL-tinaja.....neg

'La tinaja no es tuya'

*'Vos no sos una tinaja'

En (20a-c) las oraciones están formadas por un verbo $i h i$ 'estar/haber', un sustantivo, lape 'puerta', lhey 'nombre' y lajlap'ut 'tapa' respectivamente, que funciona como argumento del verbo 'haber' (el mismo caso, sin negación, véase en (14b)) y el afijo de negación. Se trata de oraciones existenciales negativas donde la posesión es un significado derivado de la existencia ("no hay X para Y"= 'Y no tiene X'). En (20d-f), sin embargo, no hay elemento existencial alguno (verbo $i h i$ ) sino una construcción nominal que predica de manera negativa la posesión. (20d-e) están formadas por la raíz y el prefijo pronominal posesivo, más el morfema negativo discontinuo $h a . . . . . . h i$. En (20d y e) los sustantivos son inalienables y el prefijo de posesión es obligatorio, pero en (20f) el sustantivo tuwej 'tinaja' es alienable y entonces la construcción nominal debe recibir el clasificador posesivo $k a$ - unido a la raíz tuwej 'tinaja' y al prefijo pronominal posesivo de segunda persona 
$a$-. Las glosas de los ejemplos (20d-f) demuestran además que no es posible interpretar estos casos como predicados de existencia negativos porque en ellas no se niega al referente sino a la relación de posesión que se establece entre el poseedor (indicado por el prefijo posesivo) y el poseído (el sustantivo).

Como indican (19) y (20) la negación señala al elemento que funciona como predicado en una oración. Ese predicado puede ser verbal o nominal. Si es nominal, ha...hi niega la existencia del referente (20a-c) o la relación de posesión entre el referente denotado por el sustantivo y poseedor. Sin embargo, y a pesar de que en wichí tanto verbos como sustantivos admiten la misma forma negativa, ha...hi niega exclusivamente predicados nominales posesivos. La negación entonces, distingue la función de argumento versus predicado en el caso de los sustantivos inalienables, porque éstos nunca funcionan como predicados, a menos que estén negados. Cuando son alienables pueden funcionar como predicados independientemente de la negación; no obstante, sabemos de qué clase de palabra se trata por la presencia del clasificador posesivo. De esta manera, la negación sirve a la diferenciación entre ambas clases de palabras.

\section{CONCLUSIONES}

A lo largo de esta presentación, hemos mostrado que existen dos clases léxicas mayores, sustantivo y verbo con características bien definidas. Los sustantivos y los verbos se asemejan entre sí en tanto que el prefijo pronominal es obligatorio para todas las clases de verbos y para los sustantivos de posesión inalienable. Esto nos ha llevado a preguntarnos qué diferencia existiría entre ambas clases en los casos en que lo que figura como palabra consta de la raíz o un tema y un prefijo pronominal exclusivamente. Las posibilidades morfológicas de ambas clases, es decir las categorías y clases posicionales que podemos esperar en cada caso son muchas y diferentes. Sin embargo, vimos que la mayoría de ellas son optativas. También dimos ejemplos donde una serie de formas pueden marcar por igual sustantivos y verbos. Denominamos a esas formas "enclíticos" porque a diferencia de los afijos no se ligan a una clase determinada (o a un huésped - "host").

Luego de pasar revista a la estructura morfológica de las clases, nos detuvimos en los criterios sintácticos que nos permiten diferenciarlas. Al respecto, presentamos casos donde un sustantivo puede funcionar como predicado nominal. En tales casos puntualizamos que si bien los predicados nominales no surgen en los textos, donde los sustantivos son argumentos en el 99\% de los casos, es posible elicitarlas en ciertas condiciones de contexto, en cuyo caso son aceptables. La predicación nominal ("soy X") es morfológicamente diferente del sustantivo poseído ("mi X"), a pesar de la identidad del prefijo pronominal. Otros morfemas tienen que intervenir para que esta estructura no resulte anómala. Vimos además que la negación señala que ambas clases de palabra pueden funcionar como predicado. Cuando son predicados nominales, el sustantivo es el núcleo de la predicación posesiva; cuando se trata de un predicado verbal posesivo, el núcleo es el verbo y el sustantivo funciona como argumento. En conclusión, la lengua wichí exhibe mecanismos que parecen señalar que la diferencia entre las clases mayores es robusta, sobre la base de criterios morfológicos y sintácticos. 


\section{REFERENCIASBIBLIOGRÁFICAS}

BRAUNSTEIN, J. (1992-93). Territorio e historia de los narradores matacos. Hacia una Nueva Carta Étnica del Gran Chaco V: 4-74.

CRISTAL, D. (1997). A Dictionary of Linguistics and Phonetics. London: Blackwell Publishers.

DIXON, R.M.W \& Alexandra Aikhenvald. (2002). Word: a cross-linguistic typology. Cambridge: Cambridge University Press.

GARCÍA, M.A. (2002). Paisajes sonoros de un mundo coherente. Prácticas musicales y religión en la sociedad wichí. Tesis de doctorado. Facultad de Filosofía y Letras. Universidad de Buenos Aires. (Inédito).

GOLlUSCIO, L. (1993). Clases de sustantivos y sistema cultural: la posesión en wichí. Signo y Seña $3: 221-239$.

VENDLER, Z. (1967). Linguistics and Philosophy. Ithaca: Cornell University Press.

VOGEL, P. \& COMRIE, B. (eds.) (2000). Approaches to the Typology of Word-Classes. Berlin: Mouton de Gruyter.

Recibido: 28/01/2005

Versão Corrigida: 27/07/2005

Aceito: 30/08/2005 